\title{
Solutions of the spherically symmetric SU(2) Einstein-Yang-Mills equations defined in the far field
}

\author{
Arthur G. Wasserman \\ Department of Mathematics, University of Michigan, Ann Arbor, Michigan 48109-1109
}

(Received 14 March 2000; accepted for publication 20 June 2000)

It is shown analytically that every static, spherically symmetric solution to the Einstein-Yang-Mills equations with SU(2) gauge group that is defined in the far field has finite ADM mass. Moreover, there can be at most two horizons for such solutions. The three types of solutions possible, Bartnik-McKinnon particle-like solutions, Reissner-Nordström-type solutions, and black hole solutions having only one horizon are distinguished by the behavior of the metric coefficients at the origin. (C) 2000 American Institute of Physics. [S0022-2488(00)00810-0]

\section{INTRODUCTION}

The Einstein-Yang-Mills equations with SU(2) gauge group, derived in Ref. 1 for static, spherically symmetric solutions in the magnetic ansatz, give a classical (nonquantum mechanical) description of gravity coupled to a nuclear force modeled by a Yang-Mills field. The unknowns of the equations are the metric and the connection. We may write the metric as

$$
d s^{2}=-A(r) B(r)^{-2} d t^{2}+A(r)^{-1} d r^{2}+r^{2}\left(d \theta^{2}+\sin ^{2} \theta d \phi^{2}\right),
$$

and the Yang-Mills curvature 2-form as

$$
F=w^{\prime} \tau_{1} d r \wedge d \theta+w^{\prime} \tau_{2} d r \wedge(\sin \theta d \phi)-\left(1-w^{2}\right) \tau_{3} d \theta \wedge(\sin \theta d \phi) .
$$

Here $A(r) B(r)^{-2}, A(r)^{-1}$, and $w(r)$ denote the unknown metric and connection coefficients, respectively, prime denotes the derivative with respect to $r$, the Schwarzschild coordinate, and $\left\{\tau_{1}, \tau_{2}, \tau_{3}\right\}$ form a basis for $\mathrm{su}(2)$, the Lie algebra of $\mathrm{SU}(2)$.

The EYM equations in this framework form a system of three ordinary differential equations:

$$
\begin{gathered}
r A^{\prime}+\left(1+2 w^{\prime 2}\right) A=1-W^{2} / r^{2}, \\
r^{2} A w^{\prime \prime}+r \Psi(r) w^{\prime}+w W=0, \\
r B^{\prime} / B=2 w^{\prime 2},
\end{gathered}
$$

where we set $W=1-w^{2}$ and $\Psi(r)=1-A-W^{2} / r^{2}$.

Much effort has gone into studying this system of equations-hundreds of papers have appeared. See Ref. 2 for an extensive bibliography. Much of the effort has been directed towards proving the existence of solutions of various types: particle-like solutions cf. Refs. 1, 3-6, black hole solutions cf. Refs. 7-12. Reissner-Nordström-type solutions cf. Ref. 13, "bag of gold" solutions cf. Ref. 6.

There have also been results concerning the uniqueness of solutions. For example, it is shown in Ref. 6 that particle-like solutions are classified by nodal class. Also, it is shown in Ref. 14 that any (static, spherically symmetric) solution to the EYM equations that is defined in the far field and is regular, that is, $A(r)>0$ for $r>>1$, is either a particle-like solution, a black hole solution, or a Reissner-Nordström-type solution, that is, a solution that has $A(r)>1$ for some $r$. [A solution $(A, w, B)$ is said to be defined in the far field if there is a $r_{0}>0$ such that for all $r>r_{0}$ the functions are defined and differentiable and Eqs. (1.1)-(1.3) are satisfied.] 
In this paper we consider static, spherically symmetric solutions to the SU(2) EYM equations that are defined in the far field.

Note: since Eqs. (1.1) and (1.2) do not involve $B$ we need only discuss $w$ and $A$ in what follows; Eq. (1.3) can always be solved for $B$.

The first objective is to answer a question posed in Ref. 15, are all solutions to Eqs. (1.1) and (1.2) that are defined in the far field regular solutions? That is, if a solution $(w, A)$ to $(1.1)$ and (1.2) is defined for $r>r_{0}$, is $A(r)>0$ for $r>>1$ ? The answer, given by Theorem 9, is yes. It is shown that any solution to Eqs. (1.1) and (1.2) that is defined in the far field has finite ADM mass; ${ }^{16}$ in particular, $A(r)>0$ for large $r$. Thus, any solution defined in the far field is asymptotically flat space-time.

It was shown in Ref. 15 that any regular solution to the EYM equations that is defined in the far field is actually defined for all $r>0$. Combining this result with Theorem 9 quoted above we can say that any solution to the EYM equations that is defined in the far field is actually defined for all $r>0$; we then ask about the behavior of the solution near $r=0$.

If a solution is defined in the far field and $A(r)>0$ for all $r>0$ then the solution must be either a particle-like solution or a Reissner-Nordström-type solution with a naked singularity at the origin. ${ }^{14}$ What can we say about solutions if there is a horizon, that is, if $A(\rho)=0$ for some $\rho$ ? For example, can there be horizons within horizons? Are there solutions defined for all $r \geqslant 0$ that satisfy particle-like initial conditions at $r=0$ and have $A>0$ in the far field but for which $A$ has two or more zeros?

It was shown in Ref. 15 that the zeros of $A$ are isolated and can only accumulate at $r=0$; moreover, there are at most two zeros of $A$ for $r>1$. It was shown in Ref. 17 that 0 is not an accumulation point for the zeros of $A$. In Sec. III we give a very simple argument for a sharper result: we show that $A$ can have at most two zeros. This answers another question posed in Ref. 15. Moreover, if $A$ has two zeros then it must be a Reissner-Nordström-type solution with a singularity at the origin (Proposition 22). There can also be solutions for which $A$ has exactly one zero and for these solutions we show that either $\lim _{r \rightarrow 0} A(r)=\infty$ or $\liminf _{r \rightarrow 0} A(r)=-\infty$ (Theorem 20). This confirms a conjecture of Ref. 15.

We thus have a trichotomy: for solutions defined in the far field the behavior of the solutions at $r=0$ can be of three types: (a) $\lim _{r \rightarrow 0} A(r)=1$; (b) $\lim _{r \rightarrow 0} A(r)=\infty$; or (c) $\liminf _{r \rightarrow 0} A(r)$ $=-\infty$ (Theorem 23).

Solutions of type (a) must be Bartnik-McKinnon particle-like solutions; see Proposition 22 and Theorem 3.7 of Ref. 14. Solutions of type (b) must be Reissner-Nordström-type solutions; see Theorem 6.3. ${ }^{13}$ The classic Schwarzschild solution, $w(r) \equiv \pm 1, A(r)=1-2 M / r$, is of type (c).

Solutions of type (c) with nontrivial gauge field have not been shown to exist; ReissnerNordström-type solutions with nontrivial gauge field have only been proved to exist when there is a naked singularity. ${ }^{13}$ There is some numerical evidence for the existence of Reissner-Nordströmtype solutions for which $A$ has two zeros. ${ }^{18-20}$ See also Ref. 2, p. 49. On the other hand, for any $\rho>0$ there exists a countable number of solutions to the EYM equations (1.1)-(1.3) defined in the far field, distinguished by nodal class, and having horizon $\rho .^{6,12}$ By the results of Ref. 15 such solutions are defined for all $r>0$, have nontrivial gauge field and the metric coefficient $A$ has one or two zeros. Thus, there must exist solutions to Eqs. (1.1)-(1.3) with nontrivial gauge field of type (b) or type (c) quite possibly both types of solutions occur.

The paper is organized as follows: in Sec. II it is shown that for any solution to (1.1) and (1.2) defined in the far field $A$ can have at most two zeros. In Sec. III it is shown that for any solution to (1.1) and (1.2) defined in the far field $A$ must be positive for large $r$. In Sec. IV solutions having horizons are discussed and the trichotomy theorem is presented.

The author thanks Piotr Bizon for helpful comments.

\section{ZEROS OF $A$}

In this section we show that if $(w(r), A(r))$ is any solution to the $\mathrm{SU}(2) \mathrm{EYM}$ equations that is defined in the far field, that is, for all $r>r_{0}$ for some $r_{0}$, then $A(r)$ has at most two zeroes. 
Definition: For any solution $(w, A)$ of the EYM Eqs. (1.1) and (1.2) we set $L(r)=1+A\left(1+2 w^{\prime 2}\right)-W^{2} / r^{2}$.

Note 0: We can also write $L=r A^{\prime}+2 A\left(1+2 w^{\prime 2}\right)$. It follows from (1.1) and (1.2) that the function $L$ satisfies the equation $r L^{\prime}(r)=L\left(1-2 w^{\prime 2}\right)+2\left(W^{2} / r^{2}-A\right)$.

Proposition 1: If $L\left(r_{1}\right)=0$ then $L^{\prime}\left(r_{1}\right)>0$.

Proof: If $L\left(r_{1}\right)=0$ then $r_{1} L^{\prime}\left(r_{1}\right)=2\left(W^{2} / r_{1}^{2}-A\right)$ which is clearly positive if $A<0$. If $A \geqslant 0$ we use $L=0$ to write $W^{2} / r_{1}^{2}-A=1+2 A w^{\prime 2}>0$.

Corollary 2: If $L\left(r_{1}\right)=0$ then $L(r)>0$ for $r>r_{1}$ and $L(r)<0$ for $r<r_{1}$; hence, $L(r)=0$ can have at most one solution.

Corollary 3: If $A(\tau)=0$ and $A^{\prime}(\tau)<0$ then $A(r)>0$ for all $r<\tau$.

Proof: By Note $0, L(\tau)=\tau A^{\prime}(\tau)<0$. If $A(r)=0$ for some $r<\tau$, let $\sigma$ be the largest such $r<\tau$ with $A(r)=0$. Since $A(r)>0$ for $r$ near $\tau, r<\tau$, we have $A^{\prime}(\sigma) \geqslant 0$ and hence, $L(\sigma)=\sigma A^{\prime}(\sigma) \geqslant 0$. But by Proposition $1, L(r)<0$ for all $r<\tau$ which is a contradiction.

Corollary 4: If $A(\sigma)=0$ and $A^{\prime}(\sigma)>0$ then $A(r)>0$ for all $r>\sigma$.

Proof: By Note $0, L(\sigma)=\sigma A^{\prime}(\sigma)>0$. If $A(r)=0$ for some $r>\sigma$, let $\tau$ be the smallest such $r>\sigma$ with $A(r)=0$. Since $A(r)>0$ for $r$ near $\sigma, r>\sigma$, we have $A^{\prime}(\tau) \leqslant 0$ and hence, $L(\tau)=\tau A^{\prime}(\tau) \leqslant 0$. But by Proposition $1, L(r)>0$ for all $r>\sigma$ which is a contradiction.

Corollary 5: If $A(\gamma)=0=A^{\prime}(\gamma)$ then $A(r)>0$ for all $0<r \neq \gamma$.

Proof: We first show that if $A(\gamma)=0=A^{\prime}(\gamma)$ then $A^{\prime \prime}(\gamma)>0$. Differentiating Eq. (1.1) at $\gamma$ gives $\gamma A^{\prime \prime}(\gamma)=2 W^{2} / \gamma^{3}+4 w W w^{\prime} / \gamma^{2}$. It follows easily from Eq. (1.1) that $1-W^{2} / \gamma^{2}=0$ since $A(\gamma)=0, A^{\prime}(\gamma)=0$; from Eq. (1.2) we get $w(\gamma)=0$ since $A(\gamma)=0, \Psi(r)=0$, thus $\gamma A^{\prime \prime}(\gamma)=2 / \gamma>0$. In particular, $A(r)>0$ for $r$ near $\gamma, r \neq \gamma$.

By Note $0, L(\gamma)=\gamma A^{\prime}(\gamma)=0$ since $A(\gamma)=0$. If $A(r)=0$ for some $r>\gamma$, let $\tau$ be the smallest such $r>\sigma$ with $A(r)=0$. Since $A(r)>0$ for $r$ near $\gamma, r>\gamma$, we have $A^{\prime}(\tau) \leqslant 0$ and hence, $L(\tau) \leqslant 0$. But by Proposition $1, L(r)>0$ for all $r>\gamma$ which is a contradiction.

If $A(r)=0$ for some $r<\gamma$, a symmetric argument produces a $\sigma<\gamma$ with $L(\sigma) \geqslant 0$, another contradiction.

Theorem 6: If $(w, A)$ is a solution to the Eqs. (1.1) and (1.2) defined for $0 \leqslant r_{1}<r<r_{2}$ then $A$ has at most two zeroes.

Proof: If $A(\gamma)=0=A^{\prime}(\gamma)$ for some $r_{1}<\gamma<r_{2}$ then $A(r)>0$ for all $r \neq \gamma, r_{1}<r<r_{2}$ by Corollary 5 and hence, $A$ has only one zero. Otherwise, if $A(\sigma)=0$ and $A^{\prime}(\sigma)>0$ for some $r_{1}<\sigma<r_{2}$ then by Corollary 4, $A(r)>0$ for all $r>\sigma$ so any zero of $A$ must occur for $r<\sigma$. Symmetrically, if $A(\tau)=0$ and $A^{\prime}(\tau)<0$ for some $r_{1}<\tau<\sigma$ and $\tau$ is the largest such $r$ then by Corollary 3, $A(r)>0$ for all $r<\tau$. Thus $A$ can have at most two zeroes.

Remark 7: The theorem actually shows a bit more, namely, if $A$ has two zeroes at $\tau$ and $\sigma$ say, with $A^{\prime}(\sigma)>0$ and $A^{\prime}(\tau)<0$ then $\tau<\sigma$. Thus, for example, there do not exist local solutions to Eqs. (1.1) and (1.2) that are negative for $r$ near 0, then positive for an interval of $r$ then negative.

Remark 8: It follows from Ref. 21 that the only solution to Eqs. (1.1) and (1.2) defined in the far field having $A(\gamma)=0=A^{\prime}(\gamma)$ for some $\gamma$ and $A(r)>0$ for large $r$ is the extreme ReissnerNordström solution given by $w(r) \equiv 0, A(r)=(r-1)^{2} / r^{2}$.

\section{FARFIELD BEHAVIOR}

In this section we show that any solution to Eqs. (1.1) and (1.2) that is defined in the far field has $A(r)>0$ for larger $r$. Such solutions were dubbed "regular"' in Ref. 14. An important corollary is that any solution to the spherically symmetric SU(2) Einstein-Yang-Mills equations defined in the far field has finite ADM mass. Lemmas 12 and 13 were announced earlier. ${ }^{15}$

Theorem 9: If $(w, A)$ is a solution to Eqs. (1.1) and (1.2) defined in the far field then $A(r)>0$ for large $r$.

Proof: Assume throughout this section that $(w, A)$ is a solution to equations (1.1) and (1.2) defined in the far field with $A(r)<0$ for large $r$; we will derive a contradiction.

The proof requires a number of lemmas.

Lemma 10: $L(r)>0$ for large $r$; in particular, $1-W^{2} / r^{2}>0, \Psi(r)>0$, and $-2 A w^{\prime 2}<1$. 
Proof: We calculate $r(L+A)^{\prime}=2-2 A-2 w^{\prime 2} L$. If $L<0$ and $A<0$ then $r(L+A)^{\prime} \geqslant 2$ and hence, $L+A>0$ for large $r$. Thus $L>-A>0$ for large $r$. Next, since $1+A\left(1+2 w^{\prime 2}\right)-W^{2} / r^{2}$ $>0$ it follows that $1-W^{2} / r^{2}>-A\left(1+2 w^{\prime 2}\right) \geqslant 0$. Also, $\Psi(r)=1-W^{2} / r^{2}-A>1-W^{2} / r^{2}>0$. The last assertion follows from $1+2 A w^{\prime 2}>W^{2} / r^{2}-A \geqslant 0$.

Lemma 11: $\liminf _{r \rightarrow \infty} 1-\left(W^{2} / r^{2}\right)=0$.

Proof: By Lemma 10 we have $1-\left(W^{2} / r^{2}\right)>0$ for large $r$. Suppose $\liminf _{r \rightarrow \infty} 1-\left(W^{2} / r^{2}\right)$ $=2 \eta>0$. Then for large $r, 1-W^{2} / r^{2}>\eta$. Note that $(r A)^{\prime}=1-W^{2} / r^{2}-\left(1+2 w^{\prime 2}\right) A \geqslant 1$ $-W^{2} / r^{2} \geqslant \eta$ so $r A>0$ for some $r$. That is a contradiction.

Lemma 12: The projection of the orbit $\left(w, w^{\prime}\right)$ in the $w-w^{\prime}$ plane cannot remain in the second or fourth quadrant Q2 or Q4 for all $r>r_{2}$.

Proof: In Q2 and Q4 $w w^{\prime}<0$ so $w^{2}$ is decreasing; hence, $\liminf _{r \rightarrow \infty} 1-\left(W^{2} / r^{2}\right)=1$ contradicting Lemma 11.

Lemma 13: The projection of the orbit $\left(w, w^{\prime}\right)$ in the $w-w^{\prime}$ plane cannot remain in the first or third quadrant $\mathrm{Q} 1$ or $\mathrm{Q} 3$ for all $r>r_{2}$.

Proof: In Q1 we have

$$
r\left(A+A w^{\prime}\right)^{\prime}=-2 A w^{\prime 2}-A-\frac{2 A w^{\prime 3}}{r}+1-\frac{W^{2}}{r^{2}}-\frac{w W}{r}>1-\frac{W^{2}}{r^{2}}-\frac{w W}{r} .
$$

Now $1-W^{2} / r^{2} \geqslant 0$ by Lemma 10 and hence $w<\sqrt{r+1}$. In the interval $0 \leqslant w \leqslant \sqrt{r+1}$ the (abstract) function of $w, 1-\left(W^{2} / r^{2}\right)-(w W / r)>1 / 4$ so $r\left(A+A w^{\prime}\right)^{\prime} \geqslant 1-\left(W^{2} / r^{2}\right)-(w W / r)>1 / 4$ and thus $A\left(1+w^{\prime}\right)>0$ for larger $r$. But $w^{\prime}>0$ in $\mathrm{Q} 1$ and hence $A>0$ which is a contradiction. Thus the orbit must leave Q1.

In Q3 we use

$$
r\left(A-A w^{\prime}\right)^{\prime}=+2 A w^{\prime 3}-A-\frac{2 A w^{\prime 2}}{r}+1-\frac{W^{2}}{r^{2}}+\frac{w W}{r}>1-\frac{W^{2}}{r^{2}}+\frac{w W}{r} ;
$$

the rest of the argument proceeds mutatis mutandis.

Lemmas 12 and 13 show that $A<0$ implies that the projection of the orbit $\left(w, w^{\prime}\right)$ in the $w-w^{\prime}$ plane must rotate; Eq. (1.2) shows that the rotation must be about $(1,0)$ or $(-1,0)$ or both. Lemma 11 shows that the size of the loops is unbounded.

Lemma 14: $\lim \sup _{r \rightarrow \infty} 1-\left(W^{2} / r^{2}\right)=1$.

Proof: It is clearly sufficient to show that for any $r_{0}$ there is an $r>r_{0}$ with $1-W^{2} / r^{2}=1$.

By Lemma 11 there is an $r_{1}>r_{0}$ such that $1-W^{2} / r_{1}^{2}<1 / 2$ say, i.e., $w^{2}-1>r / 2>>1$. Suppose that $\left(w\left(r_{1}\right), w^{\prime}\left(r_{1}\right)\right)$ is in Q1 (respectively, Q3). By Lemma 13 the orbit must exit to Q4 (respectively, Q2). Then, by Lemma 12, the orbit must leave Q4 (respectively, Q2); that can only happen if $w^{2}<1$ and the orbit exits to Q1 (respectively, Q3) or $w=0$ and the orbit exits to Q3 (respectively, Q1). In either case, there is an $r$ with $w(r)^{2}=1$, i.e., $1-W^{2} / r^{2}=1$.

Note that $(r A)^{\prime}=1-W^{2} / r^{2}-2 w^{\prime 2} A \geqslant 1-W^{2} / r^{2}>0$ by Lemma 10 so $\lim _{r \rightarrow \infty} r A$ exists and $\lim _{r \rightarrow \infty} r A \leqslant 0$. Henceforth, we assume there is an $M>0$ and $r_{0}$ such that $L(r)>0$, $r A(r)>-M$ for $r>r_{0}$.

To prove Theorem 9 we will show that $\int_{r_{0}}^{r_{1}} 1-\left(W^{2} / r^{2}\right) d r>M$ for some $r_{1}$ and hence,

$$
r_{1} A\left(r_{1}\right)>r_{0} A\left(r_{0}\right)+\int_{r_{0}}^{r_{1}} 1-\left(W^{2} / r^{2}\right) d r>-M+M=0 .
$$

Using Lemmas 10 and 14 we note that there are sequences $n<x_{n}<y_{n}<z_{n}$ such that $1-W^{2} / x_{n}^{2}=1, \quad 1-W^{2} / y_{n}^{2}=2 / 3, \quad 1-W^{2} / z_{n}^{2}=1 / 3$, and $w^{\prime}(r) \neq 0$ for $x_{n}<r<z_{n}$. Note that $w\left(x_{n}\right)^{2}=1, w\left(y_{n}\right)^{2}=1+\sqrt{\frac{1}{3}} y_{n} \approx \sqrt{\frac{1}{3}} y_{n} w\left(z_{n}\right)^{2}=1+\sqrt{\frac{2}{3}} z_{n} \approx \sqrt{\frac{2}{3}} z_{n}$. In particular, $w(r) \leqslant \sqrt{r}$ for $x_{n}<r<z_{n}$ and $w\left(z_{n}\right)-w\left(y_{n}\right)>\sqrt[4]{\frac{2}{3}} \sqrt{z_{n}}-\sqrt[4]{\frac{1}{3}} \sqrt{y_{n}} \approx 0.144 \sqrt{z_{n}}$. 
Now $\int_{r_{0}}^{\infty} 1-\left(W^{2} / r^{2}\right) d r>\sum_{n=1}^{\infty} \int_{x_{n}}^{z_{n}} 1-\left(W^{2} / r^{2}\right) d r$ since $1-W^{2} / r^{2}>0$ by Lemma 10 so to finish the proof of Theorem 9 it is clearly sufficient to show $\int_{x_{n}}^{z_{n}} 1-\left(W^{2} / r^{2}\right) d r$ is uniformly bounded away from 0 , that is $\int_{x_{n}}^{z_{n}} 1-\left(W^{2} / r^{2}\right) d r>\eta>0$, where $\eta$ is independent of $n$.

Now, for $x_{n}<r<z_{n}$, we have $1-W^{2} / r^{2} \geqslant 1 / 3$ so $\int_{x_{n}}^{z_{n}} 1-\left(W^{2} / r^{2}\right) d r>\int_{x_{n}}^{z_{n}} \frac{1}{3} d r=\left(z_{n}-x_{n}\right) / 3$. Thus, it is sufficient to show $z_{n}-x_{n}$ is uniformly bounded away from 0 . Clearly, if $z_{n}-x_{n}>1$ for all $n$ we are done so assume $z_{n}-x_{n}<1$. Assume also for definiteness that $w^{\prime}(r)>0$ for $x_{n}<r<z_{n}$; the argument is similar if $w^{\prime}(r)<0$. Now $w\left(x_{n}\right)=1, w\left(y_{n}\right) \approx \sqrt{y_{n}} / \sqrt{3}$, so $w\left(y_{n}\right)-w\left(x_{n}\right) \approx \sqrt{y_{n}} / \sqrt[4]{3}$. Hence,

$$
z_{n}-x_{n}>y_{n}-x_{n}=\frac{w\left(y_{n}\right)-w\left(x_{n}\right)}{w^{\prime}(\zeta)^{\prime}} \approx \frac{\sqrt{y_{n}}}{3 w^{\prime}(\zeta)}
$$

for some intermediate $\zeta, x_{n}<\zeta<y_{n}$. We now complete the proof of Theorem 9 by showing that $w^{\prime}(r) / \sqrt{r}$ is bounded for $x_{n} \leqslant r \leqslant y_{n}$. Since $z_{n}-x_{n}<1$ and $x_{n}>>1$ it follows that $\left[w^{\prime}(\zeta)\right] / \sqrt{y_{n}}$ is bounded for $x_{n} \leqslant r \leqslant y_{n}$.

Lemma 15: If $w^{\prime}(a)^{2} \geqslant 625 a$ for some $a<z_{n}$ and $x_{n}>2 M$ then $w^{\prime}(r)^{2} \geqslant 625 r$ for all $a \leqslant r$ $\leqslant z_{n}$.

Proof: Let $h(r)=w^{\prime}(r)^{2}-625 r$; then $h(a) \geqslant 0$. We show that $h(r)=0$ implies $h^{\prime}(r)>0$ and thus, $h$ can never become negative. Since $\Psi(r) \geqslant 1 / 3$ and $w(r) \leqslant \sqrt{r}$ for a $\leqslant r \leqslant z_{n}$ we have from Eq. (1.2), $\left.h^{\prime}(r)\right|_{h(r)=0}=2 w^{\prime}(r) w^{\prime \prime}(r)-625=2 w^{\prime}(r)\left\{r \Psi(r) w^{\prime}+w W\right\} /\left(-r^{2} A\right)-625 \geqslant 2 w^{\prime}(r)$ $\times\left\{r w^{\prime} / 3-r^{3 / 2}\right\} /\left(-r^{2} A\right)-625 \geqslant 50 r^{1 / 2}\left\{8 r^{3 / 2}-r^{3 / 2}\right\} /\left(-r^{2} A\right)-625 \geqslant 350 r / M-625$ where we have used $-W<r, w^{2}<r,-r A<M$. Thus, $\left.h^{\prime}(r)\right|_{h(r)=0}>0$ if $r>2 M$.

Lemma 16: $w^{\prime}(r)^{2}<625 r$ for $x_{n}<r<y_{n}$.

Proof: Suppose $w^{\prime}(a)^{2} \geqslant 625 a$ for some $a<z_{n}$, then by Lemma $15 w^{\prime}(r)^{2}>625 r$ for all $a$ $\leqslant r \leqslant z_{n}$. We now apply the estimate on $w^{\prime}$ to Eq. (1.2). First, $r \Psi(r) w^{\prime}>25 r^{3 / 2} / 3>-2 w W$. Hence, $-r^{2} A w^{\prime \prime}=\left(r-r A-W^{2} / r\right) w^{\prime}+w W \geqslant r \Psi(r) w^{\prime} / 2 \geqslant r w^{\prime} / 6$ for all $a \leqslant r \leqslant z_{n}$. Thus, by Lemma 10 ,

$$
\frac{w^{\prime \prime}}{w^{\prime 2}} \geqslant \frac{r w^{\prime}}{3 r^{2}\left(-2 A w^{\prime 2}\right)} \geqslant \frac{w^{\prime}}{3 r} \text {. }
$$

Integrating the left-hand side of the inequality from $a$ to $z_{n}$ yields

$$
\int_{a}^{z_{n}} \frac{w^{\prime \prime}}{w^{\prime 2}} d r=\frac{-1}{w^{\prime}\left(z_{n}\right)}+\frac{1}{w^{\prime}(a)} \leqslant \frac{1}{w^{\prime}(a)}
$$

Integrating the right-hand side of the inequality from $a$ to $z_{n}$ yields

$$
\int_{a}^{z_{n}} \frac{w^{\prime}}{3 r} d r>\int_{y_{n}}^{z_{n}} \frac{w^{\prime}}{3 r} d r \approx \int_{y_{n}}^{z_{n}} \frac{w^{\prime}}{3 z_{n}} d r=\frac{w\left(z_{n}\right)-w\left(y_{n}\right)}{3 z_{n}} \approx \frac{\sqrt[4]{\frac{2}{3}} \sqrt{z_{n}}-\sqrt[4]{\frac{1}{3}} \sqrt{y_{n}}}{3 z_{n}} \approx \frac{0.048}{\sqrt{z_{n}}} .
$$

Thus, $1 / w^{\prime}(a)>0.048 / \sqrt{z_{n}}$ or $w^{\prime}(a)^{2}<435 z_{n} \approx 435 a$ contradicting the assumption that $w^{\prime}(a)^{2} \geqslant 625 a$. The proof of Theorem 9 is now complete.

Corollary 17: Any solution to Eqs. (1.1) and (1.2) that is defined in the far field is defined for all $r>0$. The ADM mass of the solution is finite and $A(r) \approx 1-2 \mu / r$ for $r>>1$ where $\mu$ is the ADM mass. Moreover, either the gauge field $w \equiv 0$ or $\lim _{r \rightarrow \infty} w^{2}=1$.

Proof: Since the solution is regular $[A(r)>0$ for $r>>1]$ by Theorem 9 we may invoke the results of Ref. 14. 


\section{TRICHOTOMY THEOREM}

In this section we first consider solutions of (1.1) and (1.2) defined in the far field with $A(r)$ having exactly one zero. If $A$ has one zero, say $A(\rho)=0$, then either $A^{\prime}(\rho)=0$ or $A^{\prime}(\rho)>0$; $A^{\prime}(\rho)<0$ is not possible by Theorem 9 .

If $A(\rho)=0, A^{\prime}(\rho)=0$ then since we know by Theorem 9 that $A(r)>0$ for $r>>1$ the solution must be the extreme Reissner-Nordström solution ${ }^{21}$ (see Remark 8); since $A(r)=(r-1)^{2} / r^{2}, \lim _{r \rightarrow 0+} A(r)=+\infty$.

We now consider the other case, $A(\rho)=0, A^{\prime}(\rho)>0$ for some $\rho>0$. Thus $A(r)<0$ for $r<\rho$.

By Note $0, L(\rho)=\rho A^{\prime}(\rho)>0$ in this case.

Proposition 18: If $A^{\prime}(\rho)>0$ there is a $b<\rho$ such that $L(r)<0$ for all $r<b$.

Proof: We will first assume $L(r)>0$ for all $r \leqslant \rho$ and derive a contradiction. We have $r A^{\prime}=L-2 A\left(1+w^{\prime 2}\right)>-2 A$ and hence, $\left(r^{2} A\right)^{\prime}>0$. Hence, if $r_{1}<r_{2}<\rho, A\left(r_{1}\right)$ $<\left(r_{2}^{2} / r_{1}^{2}\right) A\left(r_{2}\right)$ so $\lim _{r_{1} \rightarrow 0+} A\left(r_{1}\right)=-\infty$. Since $L(r)<1+A$ we have a contradiction. Thus, $L(b)=0$ for some $b<\rho$ and $L(r)<0$ for all $r<b$ by Proposition 1 .

Proposition 19: $\lim _{r \rightarrow 0+} L(r)=-\infty$.

Proof: We calculate $r(L+A)^{\prime}=2-2 A-2 w^{\prime 2} L$. If $L<0$ and $A<0$ then $r(L+A)^{\prime} \geqslant 2$ and hence, by integrating, $\lim _{r \rightarrow 0+} L+A=-\infty$. Since $L<1+A$ we have $2 L<1+L+A$ and hence the result.

Theorem 20: If $(w, A)$ is a solution to Eqs. (1.1) and (1.2) defined in the far field and for which $A$ has exactly one zero then either $\lim _{r \rightarrow 0} A(r)=\infty$ or $\liminf _{r \rightarrow 0+} A(r)=-\infty$.

Proof: If $A^{\prime}(\rho)=0$ then we have seen that $\lim _{r \rightarrow 0+} A(r)=+\infty$. If $A^{\prime}(\rho)>0$ we wish to show that $\liminf _{r \rightarrow 0+} A(r)=-\infty$. We assume $0>A(r)>M$ for $r$ near 0 and derive a contradiction. First note that $r A^{\prime}(r)<-1$ for all $r<r_{1}$ is not possible; a simple integration shows that $A(r)>0$ for some $r<r_{1}$. Similarly, $r A^{\prime}(r)>1$ for all $r<r_{2}$ is not possible; again, a simple integration shows that $\lim _{r \rightarrow 0+} A(r)=-\infty$. Thus, given any $r_{1}>0$ we must have $\left|r A^{\prime}(r)\right|<1$ for some $r<r_{1}$.

We complete the proof of the theorem with the following lemma.

Lemma 21: If $1 \geqslant r A^{\prime}(r) \geqslant-1, A(r)>M$, and $r \ll 1$ then $r^{2} A^{\prime \prime}(r) \leqslant-2$.

Proof of theorem using Lemma 21: The lemma shows that if $-1 \leqslant r_{1} A^{\prime}\left(r_{1}\right) \leqslant 1$ for some $r_{1}>0$ then, by integrating the inequality $r^{2} A^{\prime \prime}(r) \leqslant-2$, there is an $r_{2}<r_{1}$ such that $r_{2} A^{\prime}\left(r_{2}\right)>1$. Moreover, if $r A^{\prime}(r)=1$ for some $r<r_{2}$ then $r\left(r A^{\prime}(r)\right)^{\prime}=r^{2} A^{\prime \prime}(r)+r A^{\prime}(r) \leqslant-2$ $+1<0$ so $r A^{\prime}(r)>1$ for all $r<r_{2}$. As noted above, $r A^{\prime}(r)>1$ for all $r<r_{2}$ is not possible so that completes the contradiction and hence, the proof of the theorem.

Proof of Lemma 21: By Proposition 19, $-L>>1$ for $r<<1$; so $-2 A w^{\prime 2}+W^{2} / r^{2}>>1$ since $1+A$ is bounded. Since $\left|r A^{\prime}(r)\right|<1$ and $1+A$ is bounded, Eq. (1.1) says that $-2 A w^{\prime 2} \approx W^{2} / r^{2}$, hence, $-2 A w^{\prime 2}>>1$ and $W^{2} / r^{2}>>1$. Also, since $A$ is bounded and $-2 A w^{\prime 2}>>1$ we see that $w^{\prime 2}>>1$. We then compute

$$
r^{2} A^{\prime \prime}(r)=4 A w^{\prime 4}+\left(2+2 A-2 \frac{W^{2}}{r^{2}}\right) w^{\prime 2}+8 w \frac{W}{r} w^{\prime}+2 A-2+4 \frac{W^{2}}{r^{2}} .
$$

We have $-\left(W^{2} / r^{2}\right) w^{\prime 2}+4\left(W^{2} / r^{2}\right)<<0$ since $\quad w^{\prime 2}>>1$. Also, $\left[2+2 A-\left(W^{2} / r^{2}\right)\right] w^{\prime 2}$ $+8 w(W / r) w^{\prime}<<0$ since $W^{2} / r^{2}>>|w W / r|$ and $w^{\prime 2}>>\left|w^{\prime}\right|$. The remaining terms are all negative and hence, $r^{2} A^{\prime \prime}(r)<-2$.

Note: we are not able to prove that limit $A=-\infty$ reflecting the fact that $A$ oscillates near $r=0$, cf. Refs. $17-20$.

We now examine the behavior of solutions $(w, A)$ of Eqs. (1.1) and (1.2) defined in the far field for which $A$ has two zeros at $\tau$ and $\sigma$ say, with $A^{\prime}(\sigma)>0$ and $A^{\prime}(\tau)<0$.

Proposition 22: A solution ( $w, A)$ to Eqs. (1.1) and (1.2) defined in the far field for which $A$ has two zeros is a Reissner-Nordström-type solution.

Proof: By Remark 7, $\tau<\sigma$ and hence $A(r)>0$ for $r$ near 0. It was shown in Ref. 14 that any solution to (1.1) and (1.2) that is defined in a neighborhood of $r=0$ and that has $A(r)>0$ near 
$r=0$ is either a Reissner-Nordström-type solution or a Bartnik-McKinnon particle-like solution. Thus, it is sufficient to show that $(w, A)$ is not a Bartnikon. As observed in Corollary 3, $L(\tau)<0$ and hence $L(r)<0$ for all $r<\tau$ by Lemma 10. But a Bartnik-McKinnon particle-like solution has $L(0)=2$ since $A(0)=1, w^{\prime}(0)=0, w^{2}(0)=1$. Thus, the solution cannot be a Bartnik-McKinnon particle-like solution and must be a Reissner-Nordström-type solution.

Note that the singularity at $r=0$ is inside the horizon at $r=\rho>\tau>0$.

We now have the trichotomy theorem.

Theorem 23: Any solution of Eqs. (1.1) and (1.2) defined in the far field is defined for all $r>0$ and has finite ADM mass. Moreover, either $\lim _{r \rightarrow 0+} A(r)=1$ and the solution is particlelike, $\lim _{r \rightarrow 0+} A(r)=+\infty$ and the solution is Reissner-Nordström-type, or $\liminf _{r \rightarrow 0+} A(r)=$ $-\infty$.

Proof: If $A(r)>0$ for all $r>0$ then by the results of Ref. 14 mentioned above the solution is either a Bartnik-McKinnon particle-like solution for which $\lim _{r \rightarrow 0+} A(r)=1$ or a ReissnerNordström-type solution for which $\lim _{r \rightarrow 0+} A(r)=+\infty .{ }^{13}$ If $A$ has one zero, then $\lim _{r \rightarrow 0+} A(r)$ $=+\infty$ or $\liminf _{r \rightarrow 0+} A(r)=-\infty$ by Theorem 20. Finally, if $A$ has two zeros then the solution is a Reissner-Nordström-type solution by Proposition 22 and hence, $\lim _{r \rightarrow 0+} A(r)=+\infty$. By Theorems 6 and 9 there are no other cases to consider.

${ }^{1}$ R. Bartnik and J. McKinnon, “Particle-like solutions of the EYM equations," Phys. Rev. Lett. 61, 141-144 (1988).

${ }^{2}$ D. V. Gal'tsov and M. S. Volkov, hep-th/9810070.

${ }^{3}$ H. P. Kunzle and A. K. M. Masood-u-Alam, "Spherically symmetric static SU(2) EYM fields," J. Math. Phys. 31, 928-935 (1990).

${ }^{4}$ J. Smoller, S. T. Yau, A. Wasserman, and J. B. McLeod, "Smooth static solutions of the Einstein/Yang-Mills equations," Commun. Math. Phys. 143, 115-147 (1991).

${ }^{5}$ J. Smoller and A. Wasserman, "Existence of infinitely many smooth, static, global solutions of the Einstein Yang/Mills equations," Commun. Math. Phys. 15, 303-325 (1993).

${ }^{6} \mathrm{P}$. Breitenlohner, P. Forgács, and D. Maison, "Static spherically symmetric solutions of the Einstein-Yang-Mills equations," Commun. Math. Phys. 163, 141-172 (1994).

${ }^{7}$ P. Bizon, “Colored black holes,” Phys. Rev. Lett. 64, 2844-2847 (1990).

${ }^{8}$ P. Bizon and O. Popp, "No hair theorem for spherical monopoles and dyons in SU(2) EYM theory," Class. Quantum Grav. 9, 193-205 (1992).

${ }^{9}$ D. V. Gal'tsov and M. S. Volkov, "Black holes in Einstein-Yang-Mills theory,” Sov. J. Nucl. Phys. 51, 1171-1181 (1990).

${ }^{10}$ D. V. Gal'tsov and M. S. Volkov, "Sphalerons in Einstein/Yang-Mills theory," Phys. Lett. B 273, $255-259$ (1991).

${ }^{11}$ G. Lavrelashvili and D. Maison, "Regular and black hole solutions of Einstein/Yang-Mills dilaton theory," Phys. Lett. B 295, 67 (1992).

${ }^{12}$ J. Smoller, A. Wasserman, and S.-T. Yau, "Existence of black-hole solutions for the Einstein-Yang/Mills equations," Commun. Math. Phys. 154, 377-401 (1993).

${ }^{13} \mathrm{~J}$. Smoller and A. Wasserman, "Reissner-Nordström-like solutions of the spherically symmetric SU(2) Einstein/YangMills equations," J. Math. Phys. 38, 6522-6559 (1997).

${ }^{14}$ J. Smoller and A. Wasserman, "Regular solutions of the Einstein-Yang-Mills equations," J. Math. Phys. 36, 4301-4323 (1995).

${ }^{15}$ J. Smoller and A. Wasserman, gr-qc/9706039.

${ }^{16}$ R. Arnowitt, S. Deser, and C. W. Misner, The dynamics of general relativity, in Gravitation, edited by L. Witten (Wiley, New York, 1962), pp. 227-265.

${ }^{17}$ M. Yu Zotov, gr-qc/9906024.

${ }^{18}$ P. Breitenlohner, G. Lavrelashvili, and D. Maison, "Mass inflation and chaotic behaviour inside hairy black holes," Nucl. Phys. B 524, 427-443 (1998).

${ }^{19}$ E. E. Donets, D. V. Galtsov, and M. Yu Zotov, “Internal structures of Einstein-Yang-Mills black holes,” Phys. Rev. D 56, 3459-3465 (1997).

${ }^{20}$ D. V. Galtsov, E. E. Donets, and M. Yu Zotov, “On singularities in non-abelian black holes,” Pis'ma Zh. Eksp. Teor. Fiz. 65, 855-860 (1997).

${ }^{21}$ J. Smoller and A. Wasserman, "Uniqueness of extreme Reissner-Nordström solutions in SU(2) Einstein-Yang/Mills theory for spherically symmetric space-time,” Phys. Rev. D 52, 5812-5815 (1995). 